\title{
Dynamics of Coarsening Foams: Accelerated and Self-Limiting Drainage
}

\author{
Sascha Hilgenfeldt, ${ }^{1,2, *}$ Stephan A. Koehler, ${ }^{1}$ and Howard A. Stone ${ }^{1}$ \\ ${ }^{1}$ Division of Engineering and Applied Sciences, Pierce Hall, Harvard University, Cambridge, Massachusetts 02138 \\ ${ }^{2}$ Faculty of Applied Physics, University of Twente, P.O. Box 217, 7500 AE Enschede, The Netherlands
}

(Received 13 July 2000)

\begin{abstract}
The evolution of a foam is determined by drainage flow of the continuous (liquid) phase and coarsening (aging) of the dispersed phase (gas bubbles). Free-drainage experiments with slow- and fast-coarsening gases show markedly different dynamics and elucidate the importance of the coupling of the two effects. Strong coarsening leads to drainage times that are shorter (accelerated drainage) and independent of the initial liquid content (self-limiting drainage). A model incorporating the physics of both drainage and diffusive coarsening shows quantitative agreement with experiment.
\end{abstract}

DOI: 10.1103/PhysRevLett.86.4704

Foams $[1,2]$ are a prime example of a multiphase "soft condensed matter" system. They have important applications in the food and chemical industries, firefighting, mineral processing, and structural material science $[2,3]$. Recent research in foams and emulsions has centered on three topics which are often treated separately, but are in fact interdependent: drainage, coarsening, and rheology; see Fig. 1. We focus here on a quantitative description of the coupling of drainage and coarsening.

Foam drainage is the flow of liquid through channels (Plateau borders) and nodes (intersections of four channels) between the bubbles, driven by gravity and capillarity [5-7]. The foam drainage equation models the dynamics of the liquid volume fraction $\epsilon$ in the foam on length scales larger than the bubble size. The exact form of this equation depends on the mobility of liquid-gas interface and thus on the choice of surfactant [8]. Rigid interfaces result in the channel-dominated model [6,9], where viscous dissipation of the flow occurs in all of the liquid volume, most of which is in the channels. For mobile interfaces, dissipation in the nodes can dominate [10]. Both models can be treated as limiting cases of a generalized theory [11].

Foams evolve towards thermodynamic equilibrium by reducing their total surface area as the average size of the bubbles grows over time, or coarsens, by either rupture of the liquid films between bubbles or growth through diffusive exchange of gas. The gas exchange is only appreciable through the thin, almost flat film areas of the polyhedral bubbles in a dry foam. We concentrate on diffusive coarsening, as rupture can be minimized using a surfactant that generates stable films.

Previously $[10,11]$ we have minimized coarsening in order to study drainage alone. In general, however, the time scales of diffusive coarsening and drainage are not well separated, e.g., foams with small bubbles tend to coarsen quickly and drain slowly. Also, foams with gases of high solubility and diffusivity coarsen rapidly. We choose $\mathrm{C}_{2} \mathrm{~F}_{6}$, an almost insoluble gas, and highly soluble $\mathrm{CO}_{2}$, to create initially identical aqueous foams in order to study the effects of coarsening on drainage.
PACS numbers: $82.70 . \mathrm{Rr}, 47.55 . \mathrm{Mh}, 66.10 .-\mathrm{x}, 68.03 .-\mathrm{g}$

We revisit the classic free-drainage experiment that has been in use for over 40 years [12]. A vertical foam column of height $H$ with uniform volume fraction $\epsilon_{0}$ at time $t=0$ drains liquid that accumulates at the bottom with height $h(t)=H \epsilon_{0}-\int_{-H}^{0} \epsilon(z, t) d z$ (see the inset in Fig. 2a). The liquid height increases until all liquid has drained out of the foam, so that $h(t \rightarrow \infty) \equiv h_{\infty}=\epsilon_{0} H$.

To generate the foam, gas (either $\mathrm{CO}_{2}$ or $\mathrm{C}_{2} \mathrm{~F}_{6}$ ) and a soap solution containing $0.5 \%$ SDS (sodium dodecyl sulfate) by weight in distilled water are pumped separately into a single line through a filter. The resultant coarse froth is then forced through a porous brass plug, which extrudes the final foam. Imaging bubbles at the tube wall, we measure initial bubble diameters of $0.5 \mathrm{~mm}$ with $\approx 10 \%$ polydispersity. We assume the initial bubble volume to be equal to that of polyhedral bubbles in the bulk, for which $V_{0}=$ $\delta_{V} L_{0}^{3}$ with $\delta_{V} \approx 11.3$ as a typical value. The average initial edge (channel) length of bulk bubbles is then $L_{0} \approx$ $0.014 \mathrm{~cm}$. The liquid volume fraction can be adjusted by the pumping rates for gas and liquid to yield foams as

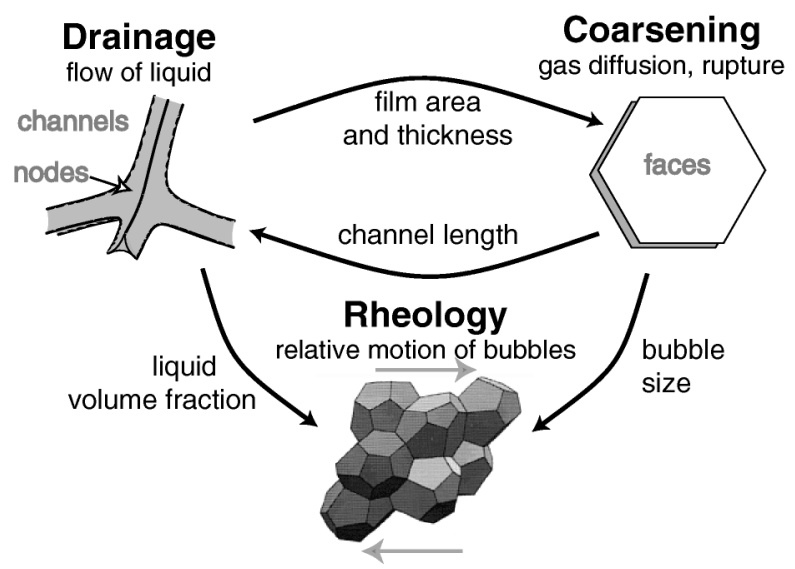

FIG. 1. Schematic of the interdependence of drainage, coarsening, and rheology of foams. For example, drainage results in a drier foam with increased shear modulus and accelerated coarsening. Coarsening in turn enhances drainage, but also decreases the shear modulus [4]. 


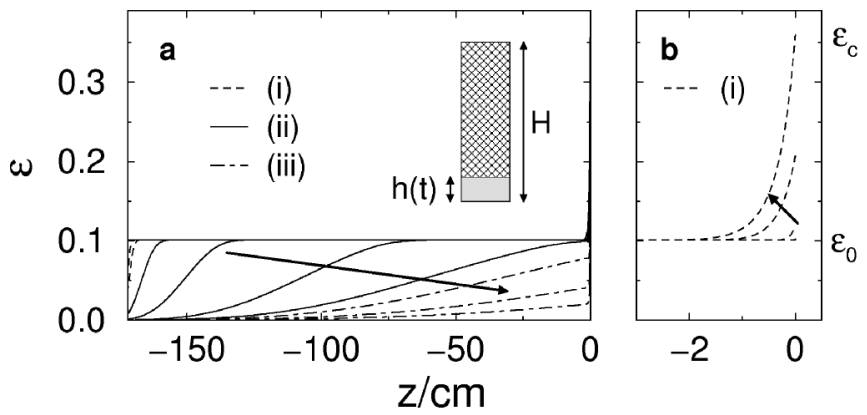

FIG. 2. Simulated free drainage of a coarsening $\mathrm{CO}_{2}$ foam with $\epsilon_{0}=0.10$ for the three stages described in the text. (a) shows the complete $\epsilon$ profiles, (b) details stage (i) for the "tail" region at the bottom, where $\epsilon(z=0)$ approaches $\epsilon_{\mathrm{c}}$. Arrows indicate increasing time; the inset in (a) sketches the accumulating liquid (gray) under the foam (hatched).

dry as $\epsilon_{0} \approx 0.01$. This apparatus was used to fill a cylinder, $172 \mathrm{~cm}$ long and $1.9 \mathrm{~cm}$ wide, with a uniform foam. During the $\approx 30 \mathrm{sec}$ of filling time, the cylinder was held horizontally to prevent drainage. Turning the cylinder vertically marks the onset of the free drainage experiment $(t=0)$ with an uncertainty of $\pm 5 \mathrm{sec}$. From chargecoupled device camera images, $h(t)$ was determined using an interpolation scheme for subpixel accuracy.

Figure 2 shows simulations, detailed below, of $\epsilon(z, t)$ for an experiment with $\mathrm{CO}_{2}(z$ points downwards with $z=0$ at the bottom of the foam). Free drainage proceeds in distinct stages: (i) Initially (dashed lines), no liquid drains out of the foam for a certain holdup time $t_{h}\left[h\left(t<t_{h}\right)=0\right]$. During this stage, $\epsilon(z=0)$ stays smaller than the liquid volume fraction of randomly close-packed spheres, $\epsilon_{\mathrm{c}} \approx 0.36$, and the foam retains liquid due to capillary suction provided by differences in curvature along the bubbles. Once $\epsilon(0)=\epsilon_{\mathrm{c}}$ is reached, the curvature differences vanish as the bubbles are spherical, and drainage begins. (ii) For $t>t_{h}$, liquid then accumulates at the bottom of the tube, and the $\epsilon=\epsilon_{0}$ plateau of the profile shrinks (solid lines). (iii) When the plateau region vanishes, the drainage flux decreases. This last stage (dot-dashed lines) can be further analyzed, but we focus on stages (i) and (ii). The experimentally observed holdup times $t_{h}$ and drainage curves $h\left(t-t_{h}\right)$ for different $\epsilon_{0}$ and different gases are shown in Figs. 3 and 4, respectively. The latter figure reveals 10-100 times faster drainage for $\mathrm{CO}_{2}$ than for $\mathrm{C}_{2} \mathrm{~F}_{6}$ and suggests power laws for $h\left(t-t_{h}\right)$ during stage (ii), with larger exponents for $\mathrm{CO}_{2}$.

To explain the measured trends, we present a model for coupling drainage and coarsening. Unlike previous approaches [13], we do not try to incorporate all possible details, but instead focus on the essential features of a theory based upon the node-dominated foam drainage equation, which we found to be a good description for the SDS foam used [11]. However, the formalism has to be modified, because $L$ is time and space dependent due to coarsening. The continuity equation for $\epsilon$ is

$$
\partial_{t} \epsilon+\partial_{z} \phi=0
$$

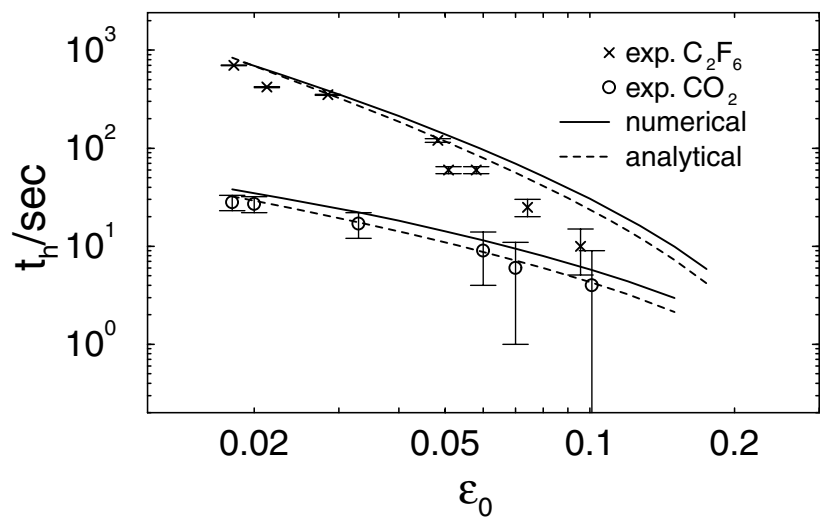

FIG. 3. Holdup times as a function of initial liquid volume fraction $\epsilon_{0}$ for $\mathrm{C}_{2} \mathrm{~F}_{6}$ and $\mathrm{CO}_{2}$ free-drainage experiments. The experimental results (symbols, with error bars) compare well with numerical simulations (solid lines) and analytical predictions [dashed lines, from Eqs. (6) and (7)].

where the (local) liquid flux $\phi(z, t)$ is given by

$$
\phi=\mu^{-1} K_{1 / 2} L^{2} \epsilon^{3 / 2}\left[\rho g+\delta_{\epsilon}^{1 / 2} \gamma \partial_{z}\left(L^{-1} \epsilon^{-1 / 2}\right)\right] .
$$

Here $\rho=1 \mathrm{~g} / \mathrm{cm}^{3}, \mu \approx 1 \mathrm{cP}$, and $\gamma \approx 30 \mathrm{~g} / \mathrm{s}^{2}$ are the density, viscosity, and surface tension of the soapy water, respectively; $\delta_{\epsilon} \approx 0.171$ is a geometrical constant, and $K_{1 / 2} \approx 0.008$ is the dimensionless permeability coefficient for node-dominated drainage whose measured values show a slight dependence on bubble size [14]. The last term in brackets of (2) is a force due to gradients in capillary pressure, inversely proportional to the radius of curvature of a channel $r=L \epsilon^{1 / 2} / \delta_{\epsilon}^{1 / 2}$. The top boundary condition for a simulation of (1) and (2) is $\phi(-H, t)=0$ for all $t$, while at the bottom $\phi(0, t)=0$ for $t<t_{h}$ and $\epsilon(0, t)=\epsilon_{\mathrm{c}}$ for $t>t_{h}$. The rising liquid reduces the total height of the foam column somewhat, but as $h_{\infty} / H$ is small, we treat $H$ as a constant in the simulations.

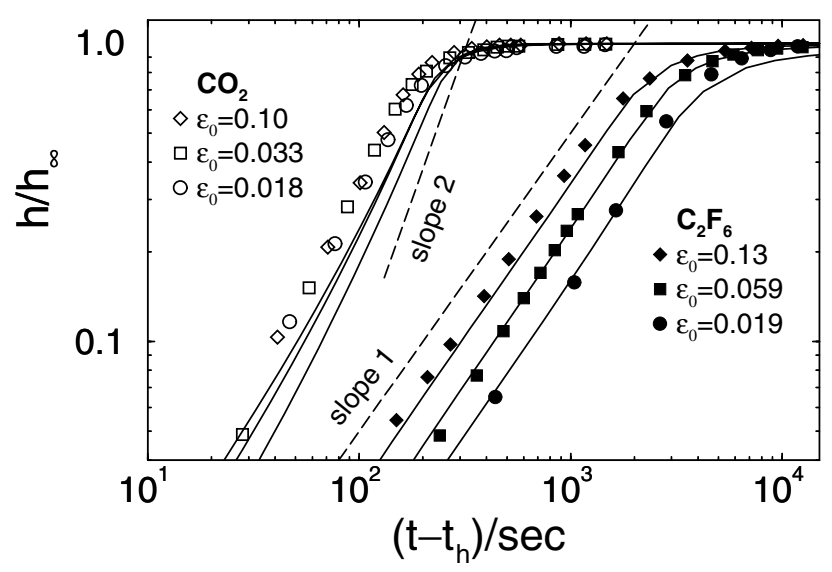

FIG. 4. Normalized drainage curves $h\left(t-t_{h}\right) / h_{\infty}$ for a $\mathrm{CO}_{2}$ foam (left data sets) and a $\mathrm{C}_{2} \mathrm{~F}_{6}$ foam (right data sets) with different $\epsilon_{0}$. Symbols are experiments, and solid lines show numerical simulations. Dashed lines indicate the expected slopes for weak (right) and ideal strong coarsening (left). Unlike the $\mathrm{C}_{2} \mathrm{~F}_{6}$ data, the drainage times for $\mathrm{CO}_{2}$ are independent of $\epsilon_{0}$. 
The coarsening of $L(z, t)$ depends on the thickness of the liquid films through which gas diffuses. Simulations of Reynolds-type film drainage equations [15] show that the films thin rapidly (typically within seconds) to black films of constant thickness $d_{\mathrm{bf}} \approx 35 \mathrm{~nm}$ [16].

The liquid volume fraction couples to the coarsening rate via $\alpha(\epsilon)$, which is the fraction of the total surface area of the bubble, $A=\delta_{A} L^{2}$, covered by thin films (this was also noted in a recent work on forced drainage [17]). Elaborate simulations of monodisperse foam structures [18] show that $\delta_{A} \approx 27$ for random dry (polyhedral) bubbles. The effective film area shrinks for wetter foams by the area occupied by the Plateau borders; following [19], we find $\alpha(\epsilon) \approx\left(1-k_{\alpha} \epsilon^{1 / 2}\right)^{2}$. The constant $k_{\alpha} \approx 1.52$ is obtained from fits to simulations of wet foam geometry with $\epsilon \lesssim 0.2$ [18]. The rate of gas exchange between bubbles is proportional to the capillary pressure difference $4 \gamma \mathcal{H}$, where $\mathcal{H}$ is the mean curvature of the face separating two bubbles. This curvature depends on the number of faces of the bubble, giving rise to a statistical analog of the (2D) von Neumann law [16,20-22]. Using bubble growth rates from this analog [22] and distributions of bubble geometry from [23], a net growth of the average bubble results corresponding to an effective mean curvature $\mathcal{H} \approx 1 /(\beta L)$ with $\beta \sim 10$ for dry foams.

With these definitions, the growth of the average bubble edge length $L(z, t)$ is described by

$$
\partial_{t} L=D_{\mathrm{eff}} \alpha(\varepsilon) / L \quad \text { with } \quad D_{\mathrm{eff}}=\frac{4 \delta_{A}}{3 \delta_{V} \beta} \frac{D_{\mathrm{f}} \mathrm{He} \gamma v_{m}}{d_{\mathrm{bf}}},
$$

where the effective diffusion coefficient $D_{\text {eff }}$ contains the ideal gas molar volume $v_{m}$, Henry's law constant $\mathrm{He}$, and gas diffusivity through the film $D_{\mathrm{f}}$. The latter can differ from the bulk value $D$ [24]. Values of $\mathrm{He}$ and $D$ are given in Table I. For long times, Eq. (3) yields $L(t) \propto t^{1 / 2}$, in agreement with experiments $[16,26]$ and earlier theory [21] of diffusive coarsening.

Inferring $D_{\text {eff }}$ directly from measurements of $L(z, t)$ in the plateau region of the foam (where $L$ is almost independent of $z$ ), we find rough agreement with Eq. (3) assuming $D_{\mathrm{f}} \approx D$; see Table I. To account for the time required to fill the foam cylinder in the experiment, we let $L$ coarsen according to (3) for $30 \mathrm{sec}$ before coupling to the foam drainage equation, so that for dry $\mathrm{CO}_{2}$ foams the effective $L_{0}$ has grown to $\approx 0.03 \mathrm{~cm}$. Estimates suggest that the filling time is also long enough to allow the liquid to saturate with the gas. Solving (1)-(3) then yields the numerical results in Figs. 2-4.
During stage (i), $\epsilon(0)$ grows from $\epsilon_{0}$ to $\epsilon_{\mathrm{c}}$. Thus, the "tail" part of the $\epsilon(z)$ profile (Fig. 2b) above the plateau $\epsilon=\epsilon_{0}$ is filled with the uniform flux $\phi_{\mathrm{p}}=$ $K_{1 / 2} L^{2} \epsilon_{0}^{3 / 2} \rho g / \mu$; cf. (2) and a similar argument in [9]. At $t=t_{h}$, and for $\epsilon_{0}$ not too large, the tail can be approximated by the steady-state profile for a stationary foam [19], which obeys the same bottom boundary conditions [zero flux and $\epsilon(0)=\epsilon_{\mathrm{c}}$ ],

$$
\epsilon_{\mathrm{s}}(z)=\epsilon_{\mathrm{c}} /\left(1-\delta_{\epsilon}^{-1 / 2} \rho g L \epsilon_{\mathrm{c}}^{1 / 2} z / \gamma\right)^{2} .
$$

The steady-state profile intersects the $\epsilon=\epsilon_{0}$ plateau at $z_{h}$ : $\epsilon_{\mathrm{s}}\left(z_{h}\right)=\epsilon_{0}$. At time $t_{h}$, the accumulated flux equals the amount of initially missing liquid in the tail,

$$
\int_{0}^{t_{h}} \phi_{\mathrm{p}} d t=\int_{0}^{z_{h}}\left[\epsilon_{\mathrm{s}}(z)-\epsilon_{0}\right] d z .
$$

In Fig. 3, the holdup times for both gases decrease with increasing $\epsilon_{0}$ due to the increased flux and the smaller amount of missing liquid in the tail. However, the holdup times for $\mathrm{CO}_{2}$ are much shorter than those for $\mathrm{C}_{2} \mathrm{~F}_{6}$, because $\phi_{\mathrm{p}}$ grows over time due to coarsening.

We use the time scale $\tau_{\mathrm{c}}=L_{0}^{2} /\left[2 D_{\text {eff }} \alpha\left(\epsilon_{0}\right)\right]$, see (3), to quantify the importance of coarsening. For $\mathrm{C}_{2} \mathrm{~F}_{6}$ and $\mathrm{CO}_{2}$, we have $\tau_{\mathrm{c}} \sim 10^{4}$ and $\sim 100 \mathrm{sec}$, respectively. Weak, moderate, or strong coarsening in region (i) ensues when $\tau_{\mathrm{c}}$ is much greater than, comparable to, or much smaller than $t_{h}$. If $\tau_{\mathrm{c}} / t_{h} \gg 1$ (weak coarsening), $L$ stays approximately constant, and we obtain from (2), (4), and (5)

$$
t_{h}^{(w)} \approx \frac{\delta_{\epsilon}^{1 / 2} \gamma \mu}{K_{1 / 2} \rho^{2} g^{2} L_{0}^{3}} \frac{\left(\epsilon_{\mathrm{c}}^{1 / 2}-\epsilon_{0}^{1 / 2}\right)^{2}}{\epsilon_{\mathrm{c}}^{1 / 2} \epsilon_{0}^{3 / 2}} .
$$

This result is self-consistent $\left(\tau_{\mathrm{c}} \gg t_{h}^{(w)}\right.$ ) for $\mathrm{C}_{2} \mathrm{~F}_{6}$ for all $\epsilon_{0}$. Experimental data and numerical simulations for $\mathrm{C}_{2} \mathrm{~F}_{6}$ agree well with (6); see Fig. 3. For dry $\mathrm{CO}_{2}$ foams, $\tau_{\mathrm{c}} \sim t_{h}$ (moderate coarsening), so that (6) is not sufficient. Treating $L$ as $z$ independent (because the plateau is the source for drainage) but time dependent, the balance (5) yields an implicit formula for $t_{h}$,

$$
t_{h}\left[1+t_{h} / 2 \tau_{\mathrm{c}}\right]\left[1+t_{h} / \tau_{\mathrm{c}}\right]^{1 / 2} \approx t_{h}^{(w)},
$$

which uses the weak coarsening result (6). An iterative solution of (7) compares well with both experiment and numerics (see Fig. 3). For very wet foams, the agreement becomes poorer, because the actual profile deviates strongly from (4), and the holdup times become comparable to the experimental accuracy.

Coarsening becomes significant in stage (ii) when $\tau_{\mathrm{c}}$ approaches the time for drainage of the plateau region $\left(\sim H \epsilon_{0} / \phi_{\mathrm{p}}\right)$. The importance of coarsening is given by

TABLE I. Material parameters He, $D$ for the two gases used [25], effective diffusivities obtained from theory $\left(D_{\text {eff }}^{\text {th }}\right)$ and measurements $\left(D_{\text {eff }}\right)$, and initial bubble sizes $L_{0}$.

\begin{tabular}{cccccc}
\hline \hline Gas & $\mathrm{He}$ & $D$ & $D_{\text {eff }}^{\text {th }}$ & $L_{0}$ & $D_{\text {eff }}$ \\
\hline $\mathrm{C}_{2} \mathrm{~F}_{6}$ & $5.5 \times 10^{-7}$ & $8.6 \times 10^{-6}$ & $2.9 \times 10^{-8}$ & 0.014 & $2.6 \times 10^{-8}$ \\
$\mathrm{CO}_{2}$ & $3.4 \times 10^{-4}$ & $1.8 \times 10^{-5}$ & $\begin{array}{c}3.7 \times 10^{-5} \\
\mathrm{~cm}^{2} \mathrm{~s}^{-1}\end{array}$ & $\begin{array}{c}0.03 \\
\mathrm{~cm}\end{array}$ & $\begin{array}{c}2.4 \times 10^{-5} \\
\mathrm{~cm}^{2} \mathrm{~s}^{-1}\end{array}$ \\
\hline Units & $\mathrm{mol} \mathrm{m}^{-3} \mathrm{~Pa}^{-1}$ & $\mathrm{~cm}^{2} \mathrm{~s}^{-1}$ & & &
\end{tabular}


$\kappa \equiv \frac{K_{1 / 2} \rho g \epsilon_{0}^{1 / 2} L_{0}^{4}}{4 \mu H D_{\text {eff }} \alpha\left(\epsilon_{0}\right)} \begin{cases}\gg 1 & \text { weak coarsening, } \\ \sim 1 & \text { moderate coarsening, } \\ \ll 1 & \text { strong coarsening. }\end{cases}$

For $\mathrm{C}_{2} \mathrm{~F}_{6}$, weak coarsening results for all $\epsilon_{0}$, except for the driest foams in our study. Thus, $L \approx L_{0}, \phi_{\mathrm{p}} \approx$ const, and we expect the liquid height to grow linearly, $h(t)=$ $\int_{t_{h}}^{t} \phi_{\mathrm{p}} d t \propto\left(t-t_{h}\right)$. In both the experiments and the simulations "linear" drainage is observed; see Fig. 4. $\mathrm{CO}_{2}$ foams show strong coarsening in stage (ii). This means that $h\left(t-t_{h}\right)$ grows linearly for $t_{h}<t \ll \tau_{\mathrm{c}}$, however it grows quadratically for $\tau_{\mathrm{c}} \ll t$, because then $\phi_{\mathrm{p}} \propto$ $L^{2} \propto t$; see (3). The trend towards this crossover behavior from linear to quadratic is visible in Fig. 4 for $\mathrm{CO}_{2}$. Given our experimental conditions, much taller foam columns are needed to observe quadratic drainage as indicated by the dashed line on the left in Fig. 4. Numerical simulations (solid lines in Fig. 4) are in good agreement with the experiments. The slightly faster drainage observed in experiment may be due to additional coarsening by film rupture, neglected in the model. $\mathrm{CO}_{2}$ simulations without coarsening would lead to drainage curves comparable to those for $\mathrm{C}_{2} \mathrm{~F}_{6}$, i.e., about 10- to 100 -fold too slow. Taller foam columns or smaller bubbles would result in even larger discrepancies.

An important difference between weak and strong coarsening is seen comparing the drainage curves for different $\epsilon_{0}$. With $\mathrm{C}_{2} \mathrm{~F}_{6}$ the curves are well separated, and wetter foams drain faster. The $\mathrm{CO}_{2}$ curves, however, "bunch" together, showing almost no $\epsilon_{0}$ dependence. Although increased $\epsilon_{0}$ leads to faster drainage, see (2), the coarsening rate is slowed due to the reduced thin film area available for gas diffusion, represented by the factor $\alpha(\epsilon)$ in (3). Thus, the drainage becomes self-limiting: increasing the wetness of the foam does not lead to faster drainage. This result was in fact empirically observed recently [27]. Self-limiting drainage could prove valuable for applications where a constant drainage rate through a foam is desired.

We have demonstrated that coarsening has a strong influence on foam drainage for gases of large solubilities, such as $\mathrm{CO}_{2}$, and small bubble sizes (diameters $<1 \mathrm{~mm}$ ). This view is supported in other recent work [28]. Strong coarsening causes a pronounced acceleration of drainage and shortens the lifetime of a foam. As a countermeasure, the beer industry adds less soluble $\mathrm{N}_{2}$ gas to the $\mathrm{CO}_{2}$ in their products [7] to stabilize the foam head. In strongly coarsening foams, we observe drainage times independent of liquid content, due to the competing effects of enhanced liquid permeability and reduced gas permeability. The effective-medium model presented here yields quantitative agreement with the experiments over a wide range of governing parameters.

We are indebted to A. Kraynik for many invaluable discussions and for sharing some of his unpublished data with us. We thank A. Evans for support of this project, and D. Durian and A. Saint-Jalmes for insightful correspondence and discussions. Support from the ACS-PRF is gratefully acknowledged.
*Email address: sascha@tn.utwente.nl

[1] J. J. Bikerman, Foams (Springer, New York, 1973).

[2] Foams: Theory, Measurements and Applications, edited by R. K. Prud'homme and S. A. Khan (Marcel Dekker, New York, 1996).

[3] L. J. Gibson and M. F. Ashby, Cellular Solids (Cambridge University Press, Cambridge, 1997).

[4] A. M. Kraynik, Annu. Rev. Fluid Mech. 20, 325 (1988); A. Saint-Jalmes and D. J. Durian, J. Rheol. 43, 1411 (1999).

[5] R. A. Leonard and R. Lemlich, AIChE J. 11, 18 (1965).

[6] I. I. Gol'dfarb, K. B. Kann, and I. R. Shreiber, Fluid Dyn. 23, 244 (1988).

[7] D. Weaire and S. Hutzler, The Physics of Foams (Oxford University Press, Oxford, 2000).

[8] M. Durand, G. Martinoty, and D. Langevin, Phys. Rev. E 60, R6307 (1999); P. J. Wilde et al., in Foams and Films, edited by D. Weaire and J. Banhart (MIT-Verlag, Bremen, 1999), p. 59.

[9] G. Verbist, D. Weaire, and A. Kraynik, J. Phys. Condens. Matter 8, 3715 (1996).

[10] S. A. Koehler, S. Hilgenfeldt, and H. A. Stone, Phys. Rev. Lett. 82, 4232 (1999).

[11] S. A. Koehler, S. Hilgenfeldt, and H. A. Stone, in Foams and Films (Ref. [8]), p. 49; Langmuir 16, 6327 (2000).

[12] W. H. Jacobi, K.E. Woodstock, and C.S. Grove, Ind. Eng. Chem. Res. 48, 2046 (1956); A. Saint-Jalmes, M. U. Vera, and D. J. Durian, Eur. Phys. J. B 12, 67 (1999).

[13] G. Narsimhan and E. Ruckenstein, Langmuir 2, 494 (1986).

[14] S. A. Koehler, S. Hilgenfeldt, and H. A. Stone (unpublished).

[15] G. Narsimhan and E. Ruckenstein, in Foams: Theory, Measurements and Applications (Ref. [2]), p. 99.

[16] C. Monnereau and M. Vignes-Adler, Phys. Rev. Lett. 80, 5228 (1998).

[17] S. Hutzler and D. Weaire, Philos. Mag. Lett. 80, 419 (2000).

[18] A. Kraynik (private communication).

[19] H. M. Princen, Langmuir 2, 519 (1986).

[20] J. von Neumann, in Metal Interfaces (American Society for Metals, Cleveland, 1952), p. 108.

[21] W. W. Mullins, J. Appl. Phys. 59, 1341 (1986); J. A. Glazier, Phys. Rev. Lett. 70, 2170 (1993).

[22] S. Hilgenfeldt, A. Kraynik, S. A. Koehler, and H. A. Stone, Phys. Rev. Lett. 86, 2685 (2001).

[23] M. Anderson and G. Grest, Philos. Mag. B 59, 293 (1989).

[24] J. T. Davies and E. K. Rideal, Interfacial Phenomena (Academic Press, New York/London, 1963).

[25] Data from NIST Chemistry WebBook, NIST Standard Reference Database No. 69, edited by W. G. Mallard and P. J. Linstrom (February 2000); Zahlenwerte und Funktionen aus Physik und Chemie, edited by H. Landolt and R. Börnstein (Springer, Berlin, 1969).

[26] D. J. Durian, D. A. Weitz, and D. J. Pine, Phys. Rev. A 44, R7902 (1991).

[27] A. Saint-Jalmes and D. Durian (private communication).

[28] M. U. Vera and D. J. Durian (unpublished). 